\title{
Primary atypical lipomatous tumor/well-differentiated liposarcoma (ALT/WDL) of the breast
}

\author{
Laurence M. Briski MD ${ }^{1}$ | Deborah O. Jeffries $M^{2}$ | Julie M. Jorns $M^{1}$ (D) \\ ${ }^{1}$ Department of Pathology, University of Michigan, Ann Arbor, MI, USA \\ ${ }^{2}$ Department of Radiology, University of Michigan, Ann Arbor, MI, USA \\ Correspondence \\ Julie M. Jorns, MD, University of Michigan, Department of Pathology, Ann Arbor, MI, USA. \\ Email: jjorns@umich.edu
}

A middle-aged woman presented with a large mobile mass in her breast. She had no history of hormonal medication use and no family history of breast, ovarian, or prostate cancer. The patient complained of soreness and increase in size of the affected breast. Physical examination revealed that her breasts were asymmetric with the affected breast being 2-3 times larger than the other side. Imaging studies showed a heterogeneous mixed density mass arising in the breast with extension into the pectoralis major muscle (Figure 1).

An ultrasound-guided core needle biopsy of the mass revealed large atypical hyperchromatic stromal cells admixed with a mature adipocytic component, histologically resembling atypical lipomatous tumor/well-differentiated liposarcoma (ALT/WDL) of soft tissue. The tumor was positive for MDM2 gene amplification by fluorescence in situ hybridization (FISH), supporting the diagnosis of a primary ALT/WDL of the breast.

The patient subsequently underwent simple mastectomy which showed an approximately $28 \mathrm{~cm}$, predominantly encapsulated mass consisting of lobulated adipose tissue admixed with bands of fibrous

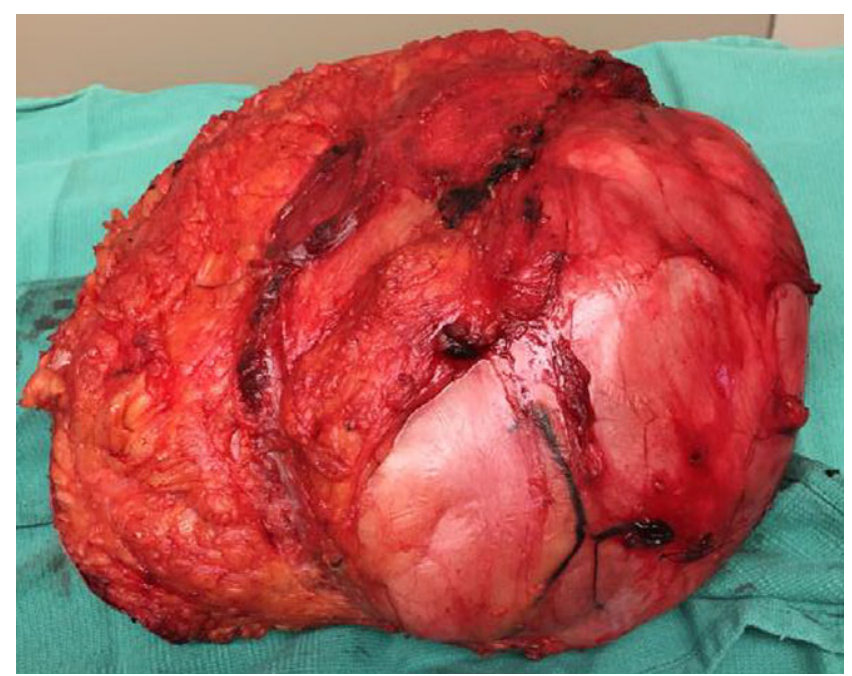

FIGURE 2 Intraoperative image of the breast mass Photo courtesy of Dr. Alfred Chang. [Color figure can be viewed at wileyonlinelibrary.com]
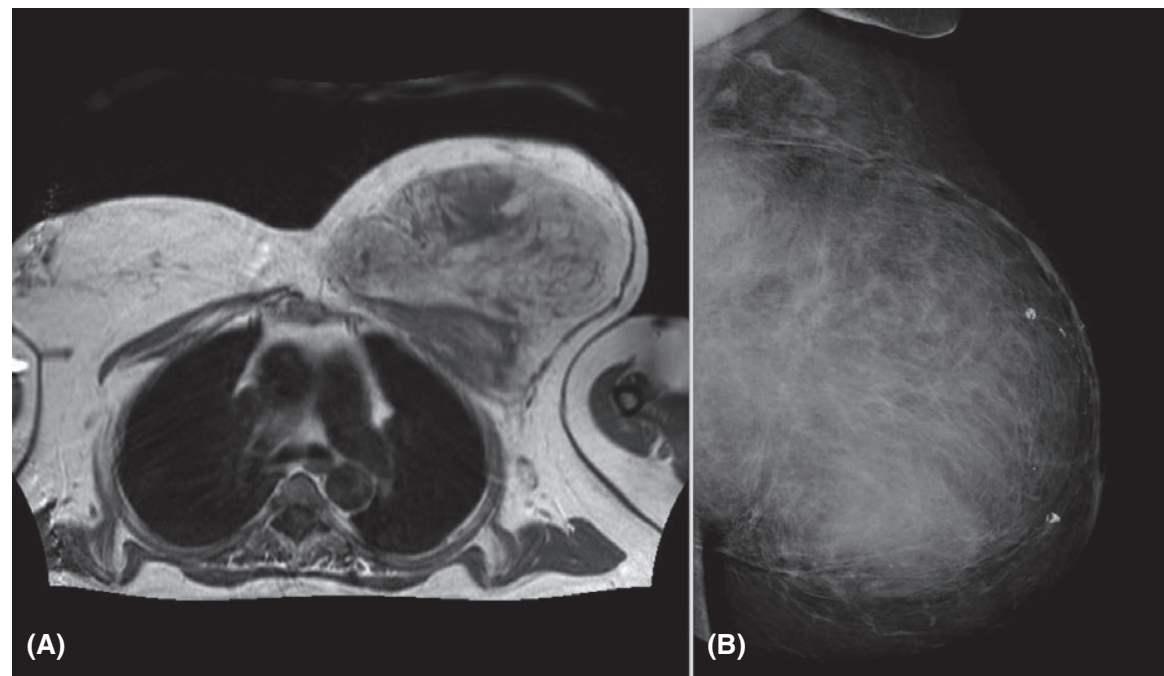

FIGURE 1 A, Axial T1-weighted image from a chest MRI showing a large heterogeneous mass arising in the breast, involving the pectoralis major and abutting the pectoralis minor. $\mathrm{B}$, Mediolateral oblique mammogram showing a heterogeneous mass containing soft tissue and fatty components 
FIGURE 3 A, Low power image of the primary breast liposarcoma (H\&E, 2X). B, High power view showing atypical stromal cells admixed with adipocytes (H\&E, 20X) and $\mathrm{C}$, corresponding MDM2 immunohistochemical stain highlighting atypical stromal cells (IHC, 10X) [Color figure can be viewed at wileyonlinelibrary.com]

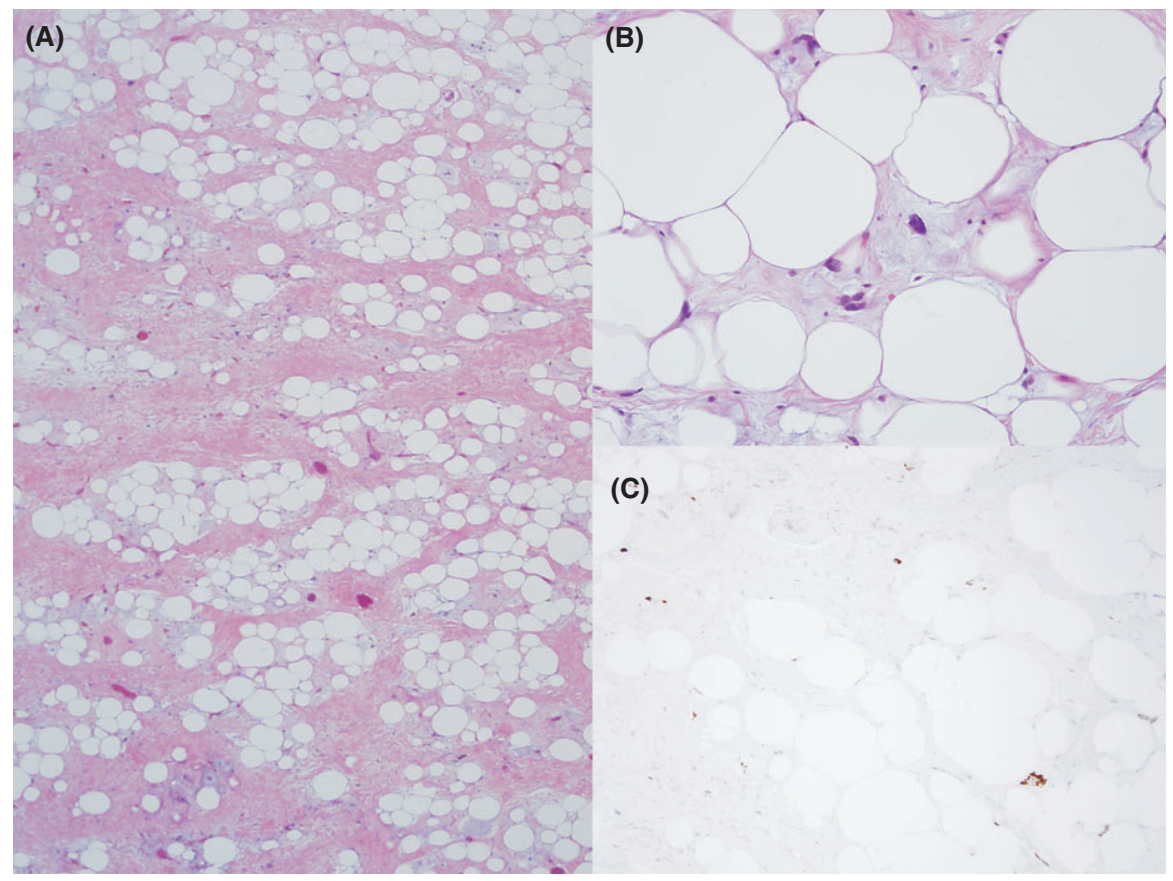

tissue, minimal fat necrosis and invasion into the underlying pectoralis major muscle (Figure 2). Histologically, the tumor consisted of mature adipose tissue admixed with bands of fibrous tissue containing characteristic large atypical hyperchromatic stromal cells consistent with a low-grade (FNCLCC grade 1) ALT/WDL. Immunohistochemical stains for CDK4 and MDM2 showed the tumor to be strongly, diffusely positive for both markers, further supporting the diagnosis of primary breast ALT/WDL (Figure 3).

Primary breast sarcomas are exceedingly rare, comprising less than $0.1 \%$ of all malignant breast tumors. The main differential diagnosis of a primary breast liposarcoma includes malignant phyllodes tumor (MPT) with liposarcomatous differentiation and, in the case of dedifferentiated liposarcoma (DDL), metaplastic breast carcinoma (MBC). Currently, all are treated relatively similarly, via surgical excision and discussion of chemotherapy. However, emerging targeted therapies may make definitive diagnosis on small samples more important. These lesions may be difficult to discern via imaging; however, they can usually be distinguished pathologically.

The main diagnostic considerations for breast tumors with a significant well-differentiated lipomatous component are MPT and primary ATL/WDL. Limited core biopsy sampling of MPT may only have sarcomatous overgrowth represented, making the two histologically indistinguishable. However, Inyang et al (Breast J. 2016. https://doi.org/10.1111/tbj.12567) and Lyle et al (Histopathology. 2016;68:1040-1045) noted that all 5 and 10 cases, respectively, of MPT with liposarcomatous stromal overgrowth lacked CDK4 and MDM2 gene amplification. This is in contrast to ATL/WDLs, which show positivity for these markers in $>90 \%$ of cases.

MBCs typically lack liposarcomatous differentiation. However, spindle cell $\mathrm{MBC}$ may be indistinguishable from DDL and both may have other heterologous sarcomatous elements such as rhabdomyo-, leiomyo-, osteo-, or chondro-sarcomatous differentiation. Unlike DDL, the majority of MBC appear to be negative for CDK4 and/or MDM2. Additionally, MBC variably express epithelial and myoepithelial markers such as cytokeratins 7, 5/6, AE1/AE3, CAM 5.2, 903 and/or MNF 116 and p63 and/or CD 10, respectively. Among these, p63 appears to be the most sensitive and specific marker for spindle cell $\mathrm{MBC}$, the main differential consideration for primary breast DDL.

\section{ORCID}

Julie M. Jorns iD http://orcid.org/0000-0002-7777-6670 